\title{
ARTICLE OPEN Field-induced double spin spiral in a frustrated chiral magnet
}

\author{
Mahesh Ramakrishnan ${ }^{1 *}$, Evan Constable ${ }^{2,3 *}$, Andres Cano $\mathbb{D}^{3,4 *}$, Maxim Mostovoy ${ }^{5}$, Jonathan S. White (D) $^{6}$, Namrata Gurung $^{4,7}$, \\ Enrico Schierle ${ }^{8}$, Sophie de Brion ${ }^{3}$, Claire V. Colin ${ }^{3}$, Frederic Gay ${ }^{3}$, Pascal Lejay ${ }^{3}$, Eric Ressouche ${ }^{9}$, Eugen Weschke ${ }^{8}$, Valerio Scagnoli $^{4,7}$, \\ Rafik Ballou (iD ${ }^{3}$, Virginie Simonet ${ }^{3}$ and Urs Staub (iD)
}

Magnetic ground states with peculiar spin textures, such as magnetic skyrmions and multifunctional domains are of enormous interest for the fundamental physics governing their origin as well as potential applications in emerging technologies. Of particular interest are multiferroics, where sophisticated interactions between electric and magnetic phenomena can be used to tailor several functionalities. We report the direct observation of a magnetic field induced long-wavelength spin spiral modulation in the chiral compound $\mathrm{Ba}_{3} \mathrm{TaFe}_{3} \mathrm{Si}_{2} \mathrm{O}_{14}$, which emerges out of a helical ground state, and is hallmarked by the onset of a unique chiralitydependent contribution to the bulk electric polarization. The periodicity of the field-induced modulation, several hundreds of nm depending on the field value, is comparable to the length scales of mesoscopic topological defects such as skyrmions, merons, and solitons. The phase transition and observed threshold behavior are consistent with a phenomenology based on the allowed Lifshitz invariants for the chiral symmetry of langasite, which intriguingly contain all the essential ingredients for the realization of topologically stable antiferromagnetic skyrmions. Our findings open up new directions to explore topological correlations of antiferromagnetic spintronic systems based on non-collinear magnetic systems with additional ferroic functionalities.

npj Quantum Materials (2019)4:60; https://doi.org/10.1038/s41535-019-0199-3

\section{INTRODUCTION}

Non-centrosymmetric magnets are outstandingly rich systems for the emergence of various types of modulated orders ${ }^{1}$ and topological objects such as skyrmions. ${ }^{2,3}$ This is related to the presence of the so-called Lifshitz invariants, as was originally demonstrated for nominal ferromagnets. ${ }^{4-6}$ Similar considerations apply to nominally collinear antiferromagnets in the absence of inversion symmetry. ${ }^{7-9}$ Thus, the popular multiferroic material $\mathrm{BiFeO}_{3}$ displays a cycloidal long-wavelength modulation of its G-type antiferromagnetic order, ${ }^{10}$ and the theoretically predicted topological defects for collinear antiferromagnets of this class ${ }^{7}$ have recently been reported in ref. ${ }^{11}$ The real challenge, however, is to generalize these concepts to non-collinear antiferromagnets, which has been motivated by numerous theoretical predictions ${ }^{12,13}$ that await experimental verification. The possibility of promoting novel magnetic textures of non-coplanar spins in non-collinear antiferromagnets is particularly appealing. Indeed, multi- $q$ objects of this type can likely display unprecedented responses and distinct topological properties. Beyond its fundamental interest, such a generalized playground could be exploited in future information technologies based on nanoscale non-collinear antiferromagnets. The potential merits of this type of antiferromagnetic-based spintronics notably includes the device-operation times, as the antiferromagnetic spin dynamics are generally much faster than their ferromagnetic counterpart. ${ }^{14,15}$ On top of that, having spintextures coupled to magnetoelectricity and/or chirality further adds the dimension of multifunctionality to the envisioned applications.

Magnetically frustrated chiral Fe-based langasites hold a lot of promise for the realization of the aforementioned topological entities. ${ }^{16}$ The langasites with chemical formula $\mathrm{Ba}_{3} \mathrm{MFe}_{3} \mathrm{Si}_{2} \mathrm{O}_{14}$
$(M=\mathrm{Sb}, \mathrm{Nb}, \mathrm{Ta})$ are multichiral systems whose crystal structure belongs to the non-centrosymmetric $P 321$ space-group symmetry, with the $\mathrm{Fe}^{3+}$ spins forming a triangular lattice in the $a b$ plane. ${ }^{16-19}$ These spins display an unconventional magnetic order that is essentially determined by frustrated exchange interactions and is peculiarly linked to the chirality of the lattice. ${ }^{16,18,20}$ Specifically, the antiferromagnetic interactions within the $\mathrm{Fe}^{3+}$ spin triangles lead to a non-collinear $120^{\circ}$ order that is helically modulated along the $c$ direction with a periodicity $\sim 7 c$ due to competing out-of-plane exchange couplings (see Fig. 1). The chirality of the $120^{\circ}$ order is determined by additional magnetocrystalline single-ion anisotropy and Dzyaloshinskii-Moriya (DM) interactions, which effectively determine the chirality of the helical modulation based on the chirality of the crystal lattice. ${ }^{19,20}$ This peculiar magnetic order enables static and dynamical magnetoelectric effects $^{21-23}$ and generates an electric polarization that can be enhanced or reversed by an applied magnetic field. ${ }^{20,24,25}$

In this article, we demonstrate the emergence of a mesoscopicscale modulation of the $120^{\circ}$ helical order in the $\mathrm{Ba}_{3} \mathrm{TaFe}_{3} \mathrm{Si}_{2} \mathrm{O}_{14}$ (BTFS) langasite. This spin texture is induced by the application of a magnetic field $\mathbf{H}$, and is signaled by an extra contribution to the electric polarization measured for $\mu_{0} H \geq 4 \mathrm{~T}$. Combining resonant $X$-ray diffraction (RXD) and neutron scattering experiments, we unveil the magnetic transition behind this singular multiferroic behavior. In accordance with the Lifshitz invariants allowed by the chiral symmetry of the langasites, the transition can be naturally explained as a continuous onset of a long-wavelength transverse conical modulation at higher fields, out of its initial $120^{\circ}$ helical ground state (double spiral). This mechanism is a generalization of previous models that were restricted to simple collinear antiferromagnets. ${ }^{7}$

\footnotetext{
${ }^{1}$ Swiss Light Source, Paul Scherrer Institut, 5232 Villigen PSI, Switzerland. ${ }^{2}$ Institute of Solid State Physics, Vienna University of Technology, 1040 Vienna, Austria. ${ }^{3}$ Univ. Grenoble Alpes, CNRS, Institut Néel, F-38042 Grenoble, France. ${ }^{4}$ Department of Materials, ETH Zurich, Vladimir-Prelog-Weg 4, 8093 Zurich, Switzerland. ${ }^{5}$ Zernike Institute for Advanced Materials, University of Groningen, Nijenborgh 4, Groningen 9747 AG, The Netherlands. ' Laboratory for Neutron Scattering and Imaging, Paul Scherrer Institut, 5232 Villigen PSI, Switzerland. ${ }^{7}$ Laboratory for Multiscale Materials Experiments, Paul Scherrer Institut, 5232 Villigen PSI, Switzerland. ${ }^{8}$ Helmholtz-Zentrum Berlin für Materialien und Energie, Wilhelm-Conrad-Röntgen-Campus BESSY II, Albert-Einstein-Strasse 15, 12489 Berlin, Germany. ${ }^{9}$ Univ. Grenoble Alpes, IRIG/DEPHY/MEM-MDN, F-38000 Grenoble, France. *email: mahesh.ramakrishnan@hotmail.com; evan.constable@tuwien.ac.at; andres.cano@neel.cnrs.fr
} 


\section{RESULTS}

Figure $2 \mathrm{a}, \mathrm{b}$ show the pyroelectric current along the $a$-axis as a function of temperature for different values of the magnetic field applied along $\mathbf{b}^{*}$ and for the two structural chiralities (see "Methods" section for experimental details). The measured current develops two clear features that are enhanced by the magnetic field. The first one signals the Néel temperature $T_{N}=28 \mathrm{~K}$ while the second peak appears at $4 \mathrm{~T}$ with a maximum at $\sim 5 \mathrm{~K}$ which shifts to $\sim 14 \mathrm{~K}$ at $8 \mathrm{~T}$. Interestingly, the relative sign of these features depends on the structural chirality, as can be seen from the comparison between Fig. 2a, b. The corresponding electric polarization is shown in Fig. 2c, d. When the two pyroelectric-

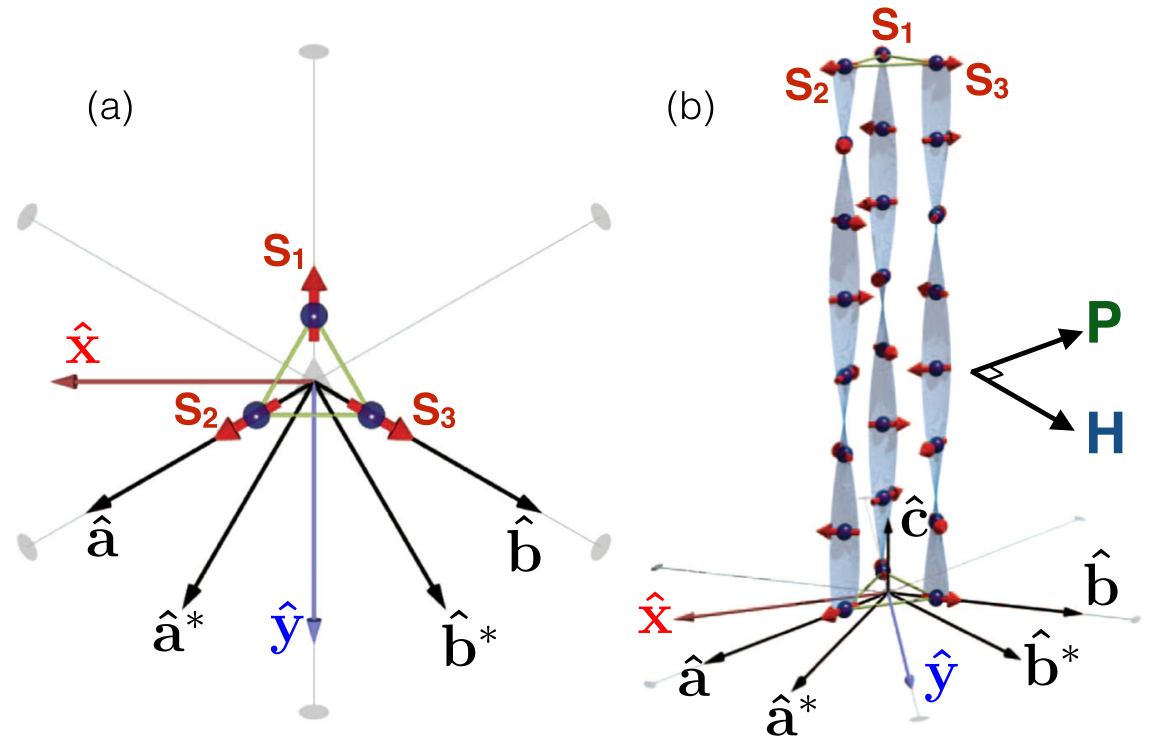

Fig. 1 Illustration of the $120^{\circ} \mathrm{Fe}^{3+}$ spin order (a) and its helical modulation along the $\mathrm{c}$ axis (b) emerging in the $\mathrm{Ba}_{3} \mathrm{MFe}_{3} \mathrm{Si}_{2} \mathrm{O}_{14}$ series in zeromagnetic field. The propagation vector is approximated to $(0,0,1 / 7)$. The different crystallographic axes, as well as the 3 -fold and 2 -fold rotation axes of the corresponding P321 space group, are indicated in (a). The direction of the electric polarization induced by a magnetic field applied along $\mathbf{b}^{*}$ is shown in (b).
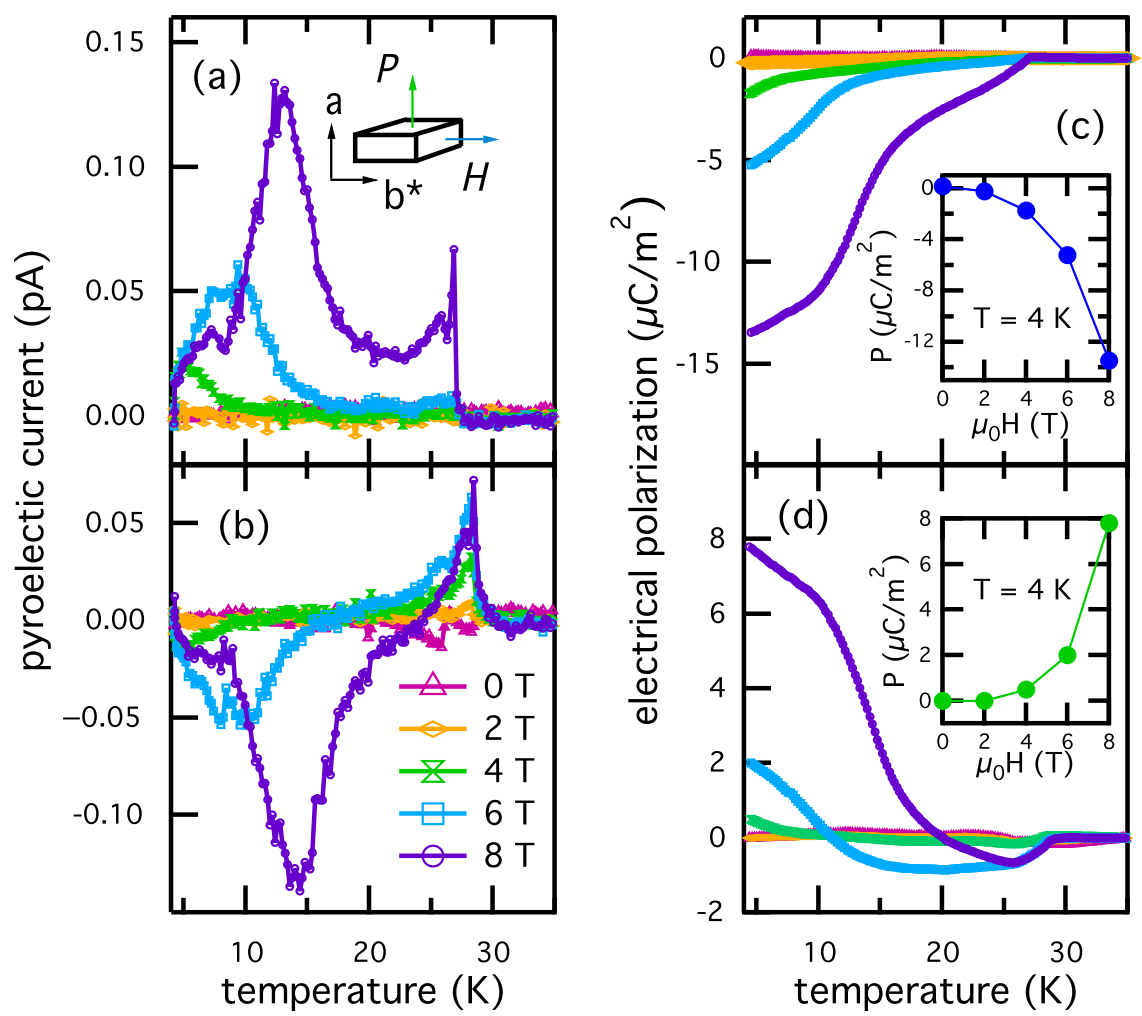

Fig. 2 a, b Temperature dependence of the pyroelectric current measured along the $a$-axis for several values of the magnetic field applied along $\mathbf{b}^{*}$. Similar results are found for $\mathbf{H} \| \mathbf{a}$ (see Supplementary Fig. 1). $\mathbf{a}, \mathbf{b}$ Correspond to two samples with opposite structural chiralities. The samples were cooled under a small poling electric field of $\sim 10 \mathrm{kV} / \mathrm{m}$. c, d Electric polarization obtained by integrating the pyroelectric data in $(\mathbf{a}, \mathbf{b})$, respectively. The insets show the electric polarization as a function of the magnetic field at $T=4 \mathrm{~K}$. 

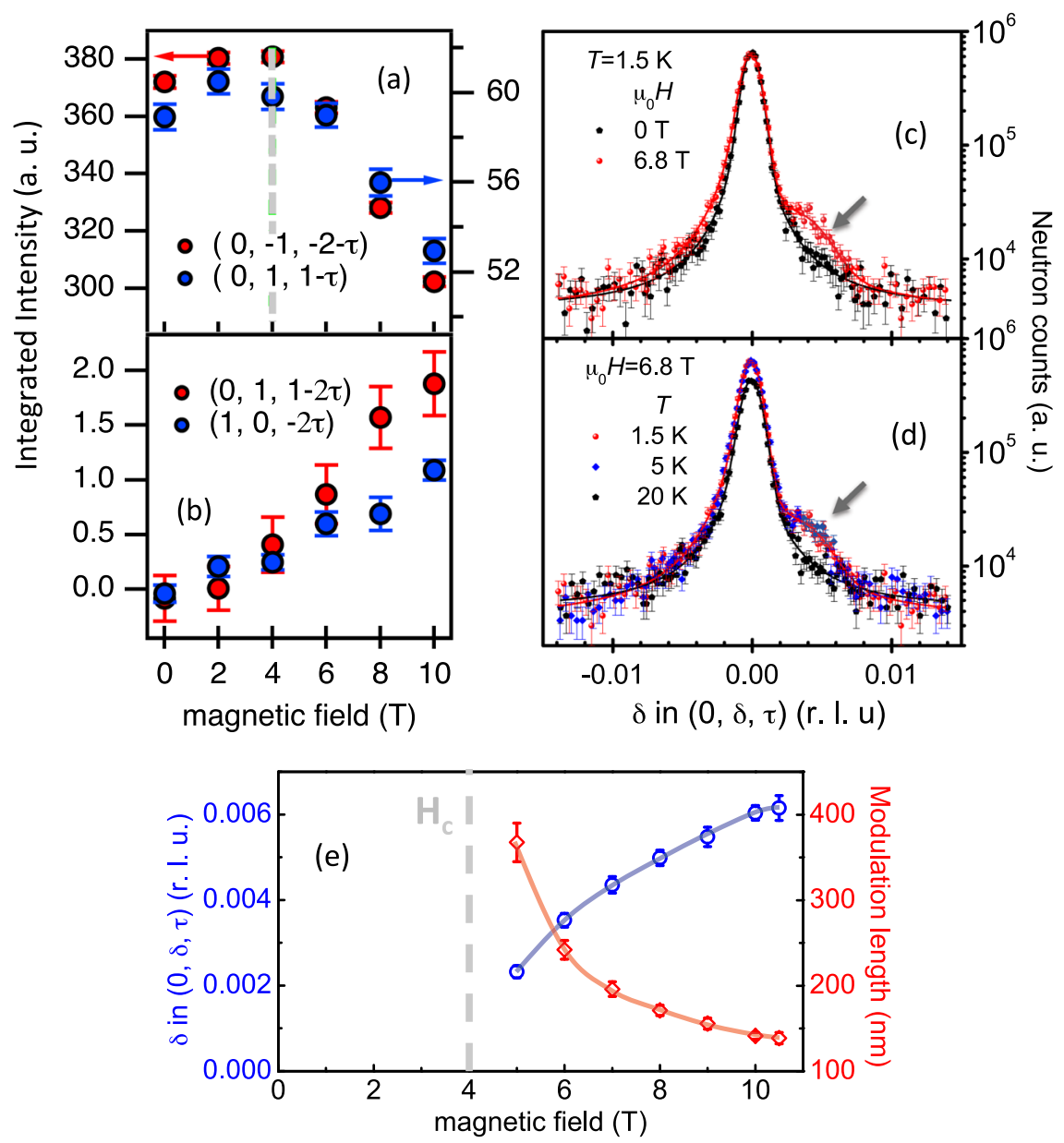

Fig. 3 a, b Field-dependence of the Bragg reflections at $\mathrm{T}=1.5 \mathrm{~K}$ measured with neutron diffraction on D23 for a magnetic field applied along the $a$-axis. Integrated intensity from rocking curves measured for first-order magnetic satellites (a), and for second-order satellites (b). c, d Rocking curves of the $(0,0, \tau)$ magnetic reflection measured on SANS-I in the $\left(\mathbf{b}^{*}, \mathbf{c}^{*}\right)$ scattering plane with a magnetic field applied along $\mathbf{b}^{*}: \mathbf{c}$ at $1.5 \mathrm{~K}$ under a magnetic field of 0 and $6.8 \mathrm{~T}$; $\mathbf{d}$ between 20 and $1.8 \mathrm{~K}$ under a magnetic field of $6.8 \mathrm{~T}$. The lines are pseudo-Voigt fits. The arrows indicate the presence of the field-induced $\delta$ satellite reflection. e Magnetic field dependence of this long-wavelength modulation wavevector extracted from pseudo-Voigt fits (left axis) and corresponding real space modulation (right axis) measured on SANS-II. The lines are a guide for the eyes.

current features have the same sign, the electric polarization displays a monotonic behavior as a function of the temperature (Fig. 2a, c). However, when the signs are opposite, the behavior is non-monotonic and the polarization is eventually reversed as in ref. ${ }^{24}$ (Fig. 2b, d). We note that the maximum component of the polarization is perpendicular to the applied magnetic field in the $a b$ plane, which is also the case if the field is applied along the a direction (see Supplementary Fig. 1).

To understand the origin of the two electric polarization signals, we looked for possible field-induced changes in the magnetic structure by neutron diffraction. Experiments were performed on the CEA-CRG D23 two-axis diffractometer at the Institut Laue Langevin (see "Methods" section for more information). We measured BTFS samples with both structural chiralities at $1.5 \mathrm{~K}$ and under a magnetic field up to $12 \mathrm{~T}$ applied along the $\mathbf{a}$ and $\mathbf{b}^{*}$ directions. We observed a rise of intensity at the zone center reflections, compatible with a ferromagnetic spin component induced along the magnetic field direction (see Supplementary Fig. 2). The intensities of the first-order magnetic satellites $(0, k$, $I \pm \tau$ ) with $\tau=0.1385$, accounting for the helical modulation along the $c$-axis, remain constant for fields up to $\approx 4 \mathrm{~T}$ before decreasing for higher fields (Fig. 3a). No change of the propagation vector was observed within the accuracy of the experiment, for any field direction and for either structural chiralities. We also followed the evolution of second-order harmonics $(0, k, I \pm 2 \tau)$, which are purely of structural origin in zero field (the structural origin is ascribed to magnetoelastic effects) (Fig. 3b). ${ }^{20}$ The intensity of several of these reflections increase slightly with increasing field due to the addition of a magnetic contribution induced by the deformation of the helix. These results are clearly indicative of a change in the magnetic structure for applied fields of over $4 \mathrm{~T}$.

To gain additional information about the new magnetic structure, complementary small-angle neutron scattering (SANS) measurements with high scattering vector $Q$-resolution were performed at the SANS-I and SANS-II instruments at SINQ, Paul Scherrer Institut (see "Methods" section for more information). The measurements were performed in the $\left(\mathbf{b}^{*}, \mathbf{c}^{*}\right)$ scattering plane under a magnetic field up to $10.5 \mathrm{~T}$ applied along $\mathbf{b}^{*}$. The field and temperature dependence of the rocking curve at the $(0,0, \tau)$ position are shown in Fig. $3 c$, d, respectively. At $1.5 \mathrm{~K}$, the $(0,0, \tau)$ satellite develops a clear shoulder when the applied field is higher than 4T. This shoulder disappears when the temperature is increased above $20 \mathrm{~K}$. This feature corresponds to a modulation of the spin structure propagating both perpendicular to the $(0,0, \tau)$ reflection and along the direction of the applied field $\mathbf{H} \| \mathbf{b}^{*}$. Remarkably, the period of such a modulation surpasses $350 \mathrm{~nm}$ at $5 \mathrm{~T}$ and decreases by increasing the applied field (see Fig. 3e). Note that a single $\delta$ satellite is observed but not its symmetric 

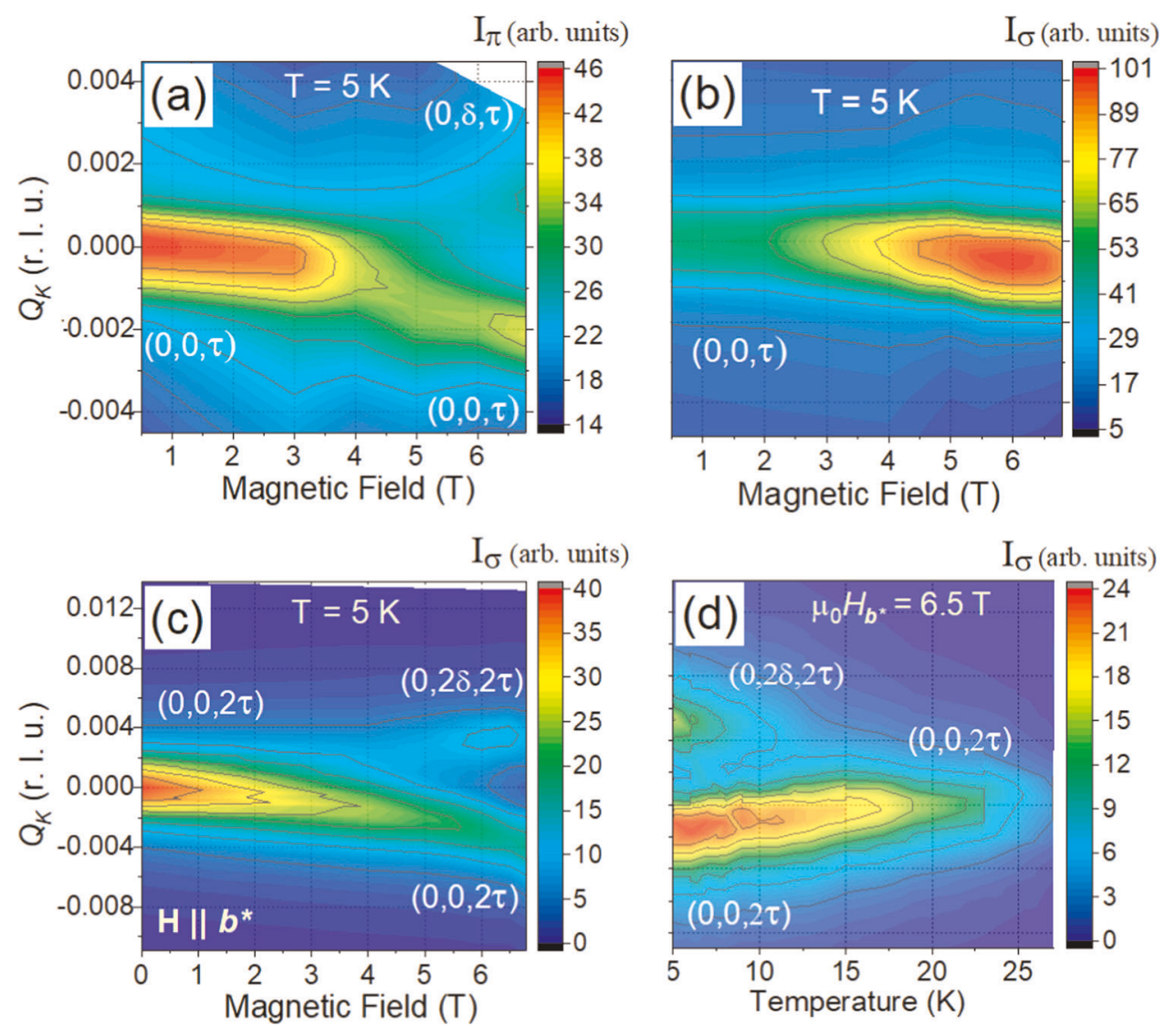

Fig. 4 a, b Dependence of the scattered intensity along $\left(0, Q_{K}, \tau\right)$ as a function of applied field along the $\mathbf{b}^{*}$ direction for (a) $\pi$ and (b) $\sigma$-polarized incident X-rays with an energy $\mathrm{E}=709.2 \mathrm{eV}$, collected at $\mathrm{T}=5 \mathrm{~K}$. The slight drift of the main reflection is an artifact caused by the small motion of the sample holder. c, $\mathbf{d}$ Scattered intensity along $\left(0, Q_{K}, 2 \tau\right)$ for $\sigma$-polarized x-rays for $\mathbf{H} \| \mathbf{b}^{*}$ : at $\mathbf{T}=5 \mathrm{~K}$ versus magnetic field (c) and at $6.8 \mathrm{~T}$ versus temperature (d). Both structures are detailed in the Supplementary Figs 7 and 8.

counterpart with respect to the main $\tau$ satellite position. This is most likely an effect of the link between structural and magnetic chirality as already observed for the main $\tau$ reflection in $\mathrm{Fe}$ langasites. ${ }^{17}$

These results are supported by RXD experiments at the $L_{3}$ edge of Fe using the high-field diffractometer on the UE46-PGM-1 beamline at the synchrotron radiation source BESSY II of the Helmoltz Zentrum Berlin. (See "Methods" for detailed information). These experiments reveal that the magnetic moments start to be noticeably affected by a magnetic field larger than 2 $T$ applied in the $a b$ plane (see Supplementary Figs 4 and 5 ). This is reflected by the change in the ratio of scattered intensity of the $(0,0, \tau)$ reflection measured with incident linear horizontal $(\sigma)$ and linear vertical $(\pi)$ X-ray polarizations, as shown in Fig. $4 a$, b for $\mathbf{H} \| \mathbf{b}^{*}$ (see additional information on the magnetic structure Supplementary Note 1 and Supplementary Fig. 3). Around 4T, this fundamental reflection $(0,0, \tau)$ then develops a shoulder, and a further increase in field strength shows a clear satellite that moves away from the main reflection for $\pi$ polarized X-rays (see Fig. 4a). This behavior gives direct confirmation for the magnetic phase transition occurring at approximately $4 \mathrm{~T}$. The additional incommensurate component, associated to $\delta$, propagates along the applied field direction and perpendicular to the zero field ordering wave vector $\boldsymbol{\tau}$. $\delta$ reaches $\approx 0.0040 \pm 0.0006$ in reciprocal lattice units at $6.8 \mathrm{~T}$, which corresponds to an incommensurate modulation with real-space periodicity of $240 \pm 40 \mathrm{~nm}$. This value, as well as its dependence on the applied field, is in good agreement with the small-angle neutron scattering experiments (see Fig. 3e).

The additional modulation of the zero-field magnetic order is also observed above a magnetic field of $4 \mathrm{~T}$ on the $(0,0,2 \tau)$ reflection (see Fig. 4c, d), which originates from the magnetic induced change in the electron density and its associated lattice deformation. Finally it can also be probed on the first-order satellite reflection at the oxygen $\mathrm{K}$-edge revealing the sensitivity of the orbital magnetic moment of the oxygen to this new spin texture (see Supplementary Fig. 6).

Note that the sensitivity of neutron scattering and RXD to the first-order magnetic reflection $(0,0, \tau)$ in zero-field is due to different deviations of the magnetic structure from the pure helical state described in Fig. 1. ${ }^{19,20,25}$ Both techniques, however probe the evolution of this reflection with magnetic field and converge to show that the magnetic structure is strongly modified in relation to the emergence of a long-wavelength modulation.

\section{DISCUSSION}

We discuss the origin of this unprecedented magnetic texture based on the parametrization introduced in ref. ${ }^{26}$ (see also ref. ${ }^{27}$ ), which uses the fact that for the $120^{\circ}$ spin order within the Fe triangles $\mathbf{S}_{1}+\mathbf{S}_{2}+\mathbf{S}_{3}=0$ and $\mathbf{S}_{1}^{2}=\mathbf{S}_{2}^{2}=\mathbf{S}_{3}^{2}$ :

$$
\left(\begin{array}{l}
\mathbf{S}_{1} \\
\mathbf{S}_{2} \\
\mathbf{S}_{3}
\end{array}\right)=\left(\begin{array}{c}
\mathbf{V}_{1} \\
-\frac{1}{2} \mathbf{V}_{1}+\frac{\sqrt{3}}{2} \mathbf{V}_{2} \\
-\frac{1}{2} \mathbf{V}_{1}-\frac{\sqrt{3}}{2} \mathbf{V}_{2}
\end{array}\right),
$$

where the vectors $\mathbf{V}_{1}=\left(X_{1}, Y_{1}, Z_{1}\right)$ and $\mathbf{V}_{2}=\left(X_{2}, Y_{2}, Z_{2}\right)$ are such that $\mathbf{V}_{1}^{2}=\mathbf{V}_{2}^{2}$ and $\mathbf{V}_{1} \cdot \mathbf{V}_{2}=0$. In our system, the frustrated exchange interactions lead to the $120^{\circ}$ order that displays a helical modulation along the $c$-axis with the wavevector $\tau=0.1385$. In addition, the spins tend to lie in the $a b$ plane due 


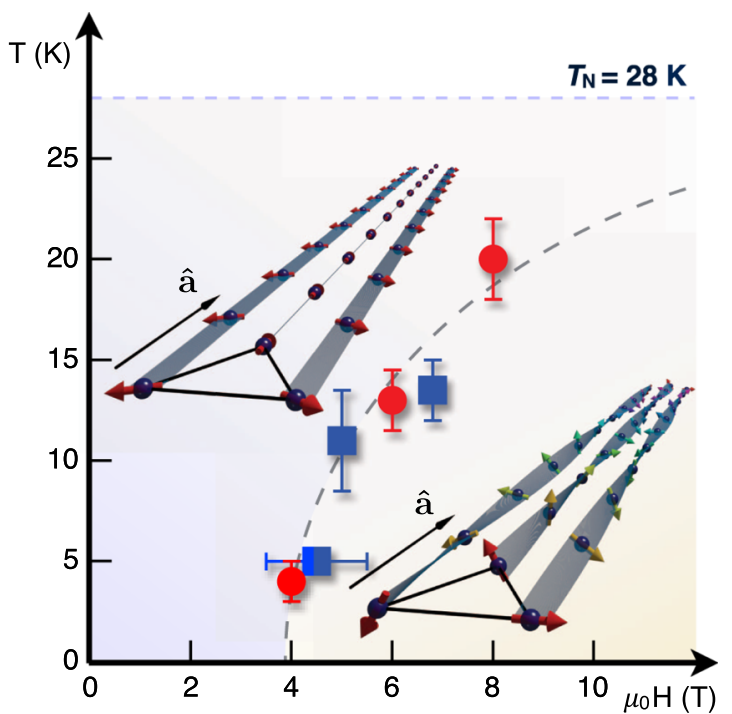

Fig. $5 \mathrm{H}-\mathrm{T}$ phase diagram of BTFS showing the field-induced mesoscopic spin texture phase. The blue squares and the red circles represent the transition points determined by RXD and pyroelectric current, respectively. They also fully agree with the SANS measurements. The insets show an oversimplified picture of the proposed zero-field and field-induced magnetic arrangements in the $a b$ plane (the field-induced ferromagnetic component is omitted for clarity). Note that the latter is drawn for a field applied along a for clarity purposes but the results are fully similar for a field along $\mathbf{b}^{*}$. In these field-induced spin structures, the plane of the $120^{\circ}$ spins rotate around the $\mathbf{b}^{*}$ (a) direction, resulting in a polarization along $\mathbf{a}\left(\mathbf{b}^{*}\right)$ for $\mathbf{H} \| \mathbf{a}\left(\mathbf{H} \| \mathbf{b}^{*}\right)$.

to the Dzyaloshinskii-Moriya interactions within the Fe triangles. ${ }^{20}$ This zero-field helical spiral is described by $\mathbf{V}_{1}=\hat{\mathbf{x}} \cos \phi+\hat{\mathbf{y}} \sin \phi$ and $\mathbf{V}_{2}=-\hat{\mathbf{x}} \sin \phi+\hat{\mathbf{y}} \cos \phi$ with $\phi=2 \pi \tau z$, where the $y$-axis corresponds to the two-fold symmetry axis along $\hat{\mathbf{a}}+\hat{\mathbf{b}}, \hat{\mathbf{x}}$ is perpendicular to the $y$-axis in the $a b$-plane, and $z$ is along the c-axis (see Fig. 1). Above a critical field $\mathbf{H} \| \mathbf{b}^{*}$, the helical spiral is expected to transform into a cycloidal one ${ }^{28}$ with $\mathbf{V}_{1}=\hat{\mathbf{z}} \cos \phi+$ $\hat{\mathbf{a}} \sin \phi$ and $\mathbf{V}_{2}=-\hat{\mathbf{z}} \sin \phi+\hat{\mathbf{a}} \cos \phi$. This final state cycloid is in the (a, c) plane that is perpendicular to $\mathbf{b}^{*}$, for $\mathbf{H} \| \mathbf{b}^{*}$.

The lack of inversion symmetry of the langasite crystal lattice allows for an additional modulation of the spiral state, which we assume to be the rotation around an axis in the $a b$-plane, $\mathbf{n}=(\cos x, \sin x, 0)$, through an angle $\psi$. The Lifshitz invariants favoring this type of modulation with an in-plane propagation vector are given in supplementary information (see symmetry analysis provided as Supplementary Note 2). Microscopically, these invariants trace back to Dzyaloshinskii-Moriya couplings in the $a b$ plane between Fe spins of different triangles not included in previous models. ${ }^{16-19,21}$ That is, combinations of antisymmetric exchange interactions of the type $\left(\mathbf{S}_{a, i} \times \mathbf{S}_{b, j}\right)_{a}$ between second neighbors $i$ and $j$ for example $(a, b=1,2,3$ and $a=x, y, z)$ that are invariants under the symmetry operations of the $P 321$ space group. In the case of a cycloidal spiral, the first three Lifshitz invariants vanish after averaging over $\phi$ since the corresponding rotation originates from symmetric exchange couplings and hence varies on a much smaller length scale than $\psi$. The fourth and fifth invariants, on the contrary, remain and become proportional to $\left[1+\sin ^{2}\left(X-\frac{\pi}{6}\right)\right](\mathbf{n} \cdot \nabla) \psi$ and $\sin \left(x-\frac{\pi}{6}\right)\left(\cos 2 x \partial_{x} \psi-\sin 2 x \partial_{y} \psi\right)$, respectively.

Thus, both these interactions favor the additional helical modulation of the spin order with a propagation vector parallel to $\mathbf{n}=\hat{\mathbf{b}}^{*}$, and hence are likely behind the field-induced spin texture observed in our system. This new non-coplanar magnetic phase can be seen as a wavering between a cycloidal and a helical state arising from an incommensurate rotation of the $120^{\circ}$ spin plane around the $\mathbf{n}$-axis perpendicular to the magnetic field direction, plus a ferromagnetic component along the field direction. A sketch of such a magnetic phase is shown in the inset of Fig. 5 (see also Supplementary Figs 7 and 8).

The energy decrease due to the additional spin rotation must overcome an increase of the anisotropy energy, which is why this rotation appears at the spiral flop transition where the anisotropy gap is reduced by the magnetic field. This scenario represents a generalization to the case of non-collinear spiral magnets of the magnetic field-induced modulations discussed in ref. ${ }^{7}$ for simple antiferromagnets and observed recently. ${ }^{29}$ The key ingredients identified here for the building of a field-induced non-coplanar mesoscopic spin texture-that is, magnetic frustration in the dominant exchange interactions supplemented by subdominant Dzyaloshinskii-Moriya couplings in a globally noncentrosymmetric setup - set the stage for the emergence of more complex objects such as skyrmions and merons in magnets of the same class under the appropriate control parameters (that is, temperature, field, pressure or strain). ${ }^{8,9,30,31}$

We are now in a position to discuss the intriguing behavior of the electric polarization $\mathbf{P}$, especially its change of sign when decreasing the temperature for one structural chirality while its sign is preserved for the other one (see Fig. 2). As described in ref. ${ }^{20}$, a first contribution to $\mathbf{P}$ below $T_{N}$ is due to the planar distortion of the initial $120^{\circ}$ order by the magnetic field, which is not compensated along the helix. This contribution, however, does not depend on the structural chirality. An additional contribution to $\mathbf{P}$ is associated to the long wavelength spin modulation, through a linear magnetoelectric effect. This effect is directly related to the absence of inversion symmetry of the lattice, and the sign of this additional contribution is defined by the sign of the crystal chirality. Such a magnetoelectric coupling, given by $-g_{1}\left(E_{x} H_{x}+E_{y} H_{y}\right)$ if the initial $120^{\circ}$ helical order preserves P321 space group symmetry elements, results in $\mathbf{P} \| \mathbf{H}$. As the field-induced spin texture breaks the two-fold axis $2_{y}$, a second magnetoelectric coupling $-g_{2}\left(E_{x} H_{y}-E_{y} H_{x}\right)$ appears. This yields $\mathbf{P} \perp \mathbf{H}$ and explains the observed chirality-dependent part of the overall electric polarization. In addition, the interaction between the magnetic order parameter $\left(\mathbf{V}_{1}, \mathbf{V}_{2}\right)$ describing the new spin texture and the electric field also results in $\mathbf{P} \perp \mathbf{H}$ (see detailed analysis provided in the Supplementary Note 2). This type of spin-driven ferroelectricity adds to other novel mechanisms observed in chiral magnetoelectrics such as those found in $\mathrm{MnSb}_{2} \mathrm{O}_{6}{ }^{32,33}$

To confirm that the new spin texture observed in the BTFS langasite is indeed behind the polarization features observed in Fig. 2, the appearance of the extra components on the $(0,0, \tau)$ and $(0,0,2 \tau)$ reflections observed by RXD was mapped out throughout the $(H, T)$ phase diagram of BTFS in Fig. 5. As we see, the onset field of the $\delta$-modulation is found to perfectly match the occurrence of the second component of the pyroelectric current and relates to the inflection points in the net polarization. We note that this transition can also be identified by an anomaly of the elastic constants. ${ }^{34}$

In summary, we have identified a field-induced long-wavelength non-coplanar magnetic texture of the spin structure of the non-collinear antiferromagnet $\mathrm{Ba}_{3} \mathrm{TaFe}_{3} \mathrm{Si}_{2} \mathrm{O}_{14}$ that is fundamentally different from those found in other chiral magnets. The new texture results in a "multi-q" antiferromagnetic order with two remarkably different wavevectors that further manifests in an additional chirality-dependent contribution to the electric polarization. The emergence of a spin texture of this type traces its origin to specific magnetic interactions that can also promote the formation of antiferromagnetic skyrmions. Thus, we anticipate that novel topological objects and exotic textures can be realized in langasites and, by analogy, in other non-centrosymmetric frustrated magnets. This potentially leads to a new topological extension of the emerging field of antiferromagnetic 
spintronics, ${ }^{14,15,35}$ based on non-collinear systems with additional multiferroic functionalities.

\section{METHODS}

The crystals were prepared and characterized as described in ref. ${ }^{21}$ The electric polarization was determined by integrating the pyroelectric current measured on slabs of BTFS single-crystals with silver pasted electrodes using a Keithley 6517A electrometer at Institut Néel. The measurements were performed in a custom split-ring superconducting cryomagnet capable of applied magnetic fields from 0 to $8 \mathrm{~T}$ and temperatures from 2 to $300 \mathrm{~K}$. Starting within the paramagnetic phase at $50 \mathrm{~K}$, the samples were cooled through the magnetic transition down to $4 \mathrm{~K}$ at a rate of $3 \mathrm{~K} / \mathrm{min}$ with a constant applied magnetic field and small $5 \mathrm{~V}$ electric bias to ensure the selection of a single domain while suppressing a parasitic zeromagnetic field signal that grows with the poling field strength. At $4 \mathrm{~K}$ the electrical bias was removed and the temperature and magnetic field were maintained for $30 \mathrm{~min}$ to allow charge build-up on the electrodes to dissipate. The sample was then heated at $3 \mathrm{~K} / \mathrm{min}$ in the same magnetic field up to $50 \mathrm{~K}$ while the pyroelectric current was measured as a function of time. The magnetic field-induced pyroelectric current was found insensitive to the direction of the poling field as reported in ref. ${ }^{24}$ Two principal samples with thicknesses of 480 and $460 \mu \mathrm{m}$ were tested (Figs 2a, $\mathrm{c}$ and $2 \mathrm{~b}, \mathrm{~d}$ of the main paper, respectively). They were chosen since they present opposite structural chiralities as determined by anomalous X-ray scattering. The measurements performed for a magnetic field $\mathbf{H} \| \mathbf{a}$ while measuring the pyroelectric current along $\mathbf{b}^{*}$ on the same sample as in Fig. 2a are shown in Supplementary Fig. 1.

The neutron diffraction experiments were performed at the Institut Laue Langevin on the CEA-CRG D23 two-axis diffractometer with a lifting arm detector and an incident wavelength of $1.2794 \AA$. The BTFS single crystal was mounted in the $12 \mathrm{~T}$ cryomagnet with the $\mathbf{a}$ or $\mathbf{b}^{*}$ direction set vertical, parallel to the applied field, and cooled down to $1.5 \mathrm{~K}$. The neutron intensities at selected Bragg positions were obtained from integration of rocking curves recorded for increasing magnetic field. An example is shown in Supplementary Fig. 2 for the $(0,0,-2)$ nuclear reflection at zerofield, which acquires a ferromagnetic component under magnetic field.

Two small-angle neutron scattering (SANS) experiments were also performed using the SANS-I and SANS-II instruments at SINQ, Paul Scherrer Institut, Switzerland. To observe the magnetic field-induced $(0, \delta, \tau)$ peaks each instrument was configured in a high resolution, high-Q setting. At SANS-I (SANS-II) a neutron wavelength of $4.7 \AA$ ( $4.8 \AA$ ) was chosen with a $10 \%$ FWHM spread and collimated over a distance of $18 \mathrm{~m}(6 \mathrm{~m})$ before the sample. Scattered neutrons were detected by a two-dimensional multidetector placed $2.8 \mathrm{~m}(1.5 \mathrm{~m})$ behind the sample. At each instrument, the sample was installed inside a horizontal field cryomagnet (6.8 T maximum field available at SANS-I, 10.5 T maximum field available at SANS-II), with the magnetic field direction first approximately aligned parallel to the neutron beam. The sample was installed inside the magnet with $\mathbf{a} \mathbf{b}^{*}-\mathbf{c}^{*}$ horizontal scattering plane. Here $\mathbf{b}^{*}$ was aligned with both the neutron beam and magnetic field leaving $\mathbf{c}^{*}$ aligned in the perpendicular direction. The SANS measurements were done by rotating the sample and magnet together around the vertical axis, and over an angular range that moved the magnetic diffraction peaks through the Bragg condition at the detector. Using the GRASP ${ }^{36}$ software, the magnetic diffracted intensity was extracted from the region of interest on the SANS detector at each rotation angle, leading to the rocking curves like those shown in Supplementary Fig. 3.

The resonant $X$-ray diffraction (RXD) experiments were carried out using the high-field diffractometer on the UE46-PGM-1 beamline at the synchrotron radiation source BESSY II. The polished and annealed BTFS sample (see ref. ${ }^{37}$ ) was mounted with the [001] direction in the horizontal scattering plane and it was cooled down to $5 \mathrm{~K}$ in a He cryomagnet. The energy dependence of the $(0,0, \tau)$ reflection in zero field is displayed in Supplementary Fig. 4. The X-ray probing depth is in the order of $50 \mathrm{~nm}$ in strong contrast to the neutron scattering experiments that probe the whole crystal. Nevertheless, results from both techniques are consistent with each other.

\section{DATA AVAILABILITY}

The datasets generated during and/or analyzed during the current study are available from the corresponding authors on reasonable request.
Received: 29 July 2019; Accepted: 8 November 2019; Published online: 04 December 2019

\section{REFERENCES}

1. Izyumov, Y. A. Modulated, or long-periodic, magnetic structures of crystals. Sov. Phys. Usp. 27, 845-867 (1984).

2. Mühlbauer, S. et al. Skyrmion lattice in a chiral magnet. Science 323, 915-919 (2009).

3. Fert, A., Reyren, N. \& Cros, V. Magnetic skyrmions: advances in physics and potential applications. Nat. Rev. Mater 2, 1-15 (2017).

4. Dzyaloshinskii, I. Theory of helicoidal structures in antiferromagnets. I. Nonmetals. Sov. Phys. JETP 19, 960-971 (1964).

5. Bak, P. \& Jensen, M. H. Theory of helical magnetic structures and phase transitions in MnSi and FeGe. J. Phys. C: Solid State Phys 13, L881-L885 (1980).

6. Bogdanov, A. N. \& Yablonskii, D. Thermodynamically stable "vortices" in magnetically ordered crystals. The mixed state of magnets. Zh. Eksp. Teor. Fiz 95, 178-182 (1989).

7. Bogdanov, A. \& Yablonskii, D. Contribution to the theory of inhomogeneous states of magnets in the region of magnetic-field-induced phase transitions. Mixed state of antiferromagnets. Sov. Phys. JETP 69, 142-146 (1989).

8. Bogdanov, A. \& Shestakov, A. Vortex states in antiferromagnetic crystals. Phys. Solid State 40, 1350-1356 (1998).

9. Bogdanov, A. N., Rößler, U. K., Wolf, M. \& Müller, K.-H. Magnetic structures and reorientation transitions in noncentrosymmetric uniaxial antiferromagnets. Phys. Rev. B 66, 214410 (2002).

10. Sosnowska, I. M. Spatially modulated spin structure (SMSS) in $\mathrm{BiFeO}_{3}-30$ years later. J. Phys.: Condens. Matter 28, 421002 (2016).

11. Seki, S., Yu, X. Z., Ishiwata, S. \& Tokura, Y. Observation of skyrmions in a multiferroic material. Science 336, 198-201 (2012).

12. Rosales, H. D., Cabra, D. C. \& Pujol, P. Three-sublattice skyrmion crystal in the antiferromagnetic triangular lattice. Phys. Rev. B 92, 214439 (2015).

13. Osorio, S. A., Rosales, H. D., Sturla, M. B. \& Cabra, D. C. Composite spin crystal phase in antiferromagnetic chiral magnets. Phys. Rev. B 96, 024404 (2017).

14. Jungwirth, T. et al. The multiple directions of antiferromagnetic spintronics. Nat. Phys. 14, 200-203 (2018).

15. Gomonay, O., Baltz, V., Brataas, A. \& Tserkovnyak, Y. Antiferromagnetic spin textures and dynamics. Nat. Phys. 14, 213-216 (2018).

16. Marty, K. et al. Single domain magnetic helicity and triangular chirality in structurally enantiopure $\mathrm{Ba}_{3} \mathrm{NbFe}_{3} \mathrm{Si}_{2} \mathrm{O}_{14}$. Phys. Rev. Lett. 101, 247201 (2008).

17. Loire, M. et al. Parity-broken chiral spin dynamics in $\mathrm{Ba}_{3} \mathrm{NbFe}_{3} \mathrm{Si}_{2} \mathrm{O}_{14}$. Phys. Rev. Lett. 106, 207201 (2011).

18. Zorko, A. et al. Role of antisymmetric exchange in selecting magnetic chirality in $\mathrm{Ba}_{3} \mathrm{NbFe}_{3} \mathrm{Si}_{2} \mathrm{O}_{14}$. Phys. Rev. Lett. 107, 257203 (2011).

19. Scagnoli, V. et al. Dzyaloshinskii-Moriya driven helical-butterfly structure in $\mathrm{Ba}_{3} \mathrm{NbFe}_{3} \mathrm{Si}_{2} \mathrm{O}_{14}$. Phys. Rev. B 88, 104417 (2013).

20. Chaix, L. et al. Helical bunching and symmetry lowering inducing multiferroicity in Fe langasites. Phys. Rev. B 93, 214419 (2016).

21. Marty, K. et al. Magnetic and dielectric properties in the langasite-type compounds: $A_{3} B \mathrm{Fe}_{3} \mathrm{D}_{2} \mathrm{O}_{14}(A=\mathrm{Ba}, \mathrm{Sr}, \mathrm{Ca} ; B=\mathrm{Ta}, \mathrm{Nb}, \mathrm{Sb} ; D=\mathrm{Ge}, \mathrm{Si})$. Phys. Rev. $B$ 81, 054416 (2010).

22. Chaix, L. et al. THz magnetoelectric atomic rotations in the chiral compound $\mathrm{Ba}_{3} \mathrm{NbFe}_{3} \mathrm{Si}_{2} \mathrm{O}_{14}$. Phys. Rev. Lett. 110, 157208 (2013).

23. Narita, H. et al. Observation of nonreciprocal directional dichroism via electromagnon resonance in a chiral-lattice helimagnet $\mathrm{Ba}_{3} \mathrm{NbFe}_{3} \mathrm{Si}_{2} \mathrm{O}_{14}$. Phys. Rev. $B$ 94, 094433 (2016).

24. Lee, N., Choi, Y. J. \& Cheong, S.-W. Magnetic control of ferroelectric polarization in a self-formed single magnetoelectric domain of multiferroic $\mathrm{Ba}_{3} \mathrm{NbFe}_{3} \mathrm{Si}_{2} \mathrm{O}_{14}$. Appl. Phys. Lett. 104, 072904 (2014).

25. Ramakrishnan, $M$. et al. Crystal symmetry lowering in chiral multiferroic $\mathrm{Ba}_{3} \mathrm{TaFe}_{3} \mathrm{Si}_{2} \mathrm{O}_{14}$ observed by x-ray magnetic scattering. Phys. Rev. B 95, 205145 (2017).

26. Reim, J. D. et al. Neutron diffraction study and theoretical analysis of the antiferromagnetic order and the diffuse scattering in the layered kagome system $\mathrm{CaBaCO}_{2} \mathrm{FeO}_{7}$. Phys. Rev. B 97, 144402 (2018).

27. Dombre, T. \& Read, N. Nonlinear $\sigma$ models for triangular quantum antiferromagnets. Phys. Rev. B 39, 6797-6801 (1989).

28. Mostovoy, M. Ferroelectricity in spiral magnets. Phys. Rev. Lett. 96, 067601 (2006).

29. Sokolov, D. A. et al. Metamagnetic texture in a polar antiferromagnet. Nat. Phys. 15, 671-677 (2019).

30. Rybakov, F. N., Borisov, A. B., Blügel, S. \& Kiselev, N. S. New type of stable particlelike states in chiral magnets. Phys. Rev. Lett. 115, 117201 (2015).

31. Kharkov, Y., Sushkov, O. \& Mostovoy, M. Bound states of skyrmions and merons near the Lifshitz point. Phys. Rev. Lett. 119, 207201 (2017). 
32. Johnson, R. D. et al. $\mathrm{MnSb}_{2} \mathrm{O}_{6}$ : A polar magnet with a chiral crystal structure. Phys. Rev. Lett. 111, 017202 (2013).

33. Kinoshita, M. et al. Magnetic reversal of electric polarization with fixed chirality of magnetic structure in a chiral-lattice helimagnet $\mathrm{MnSb}_{2} \mathrm{O}_{6}$. Phys. Rev. Lett. 117, 047201 (2016).

34. Quirion, $\mathrm{G}$. et al. Experimental evidence of symmetry breaking in the multiferroic $\mathrm{Ba}_{3} \mathrm{NbFe}_{3} \mathrm{Si}_{2} \mathrm{O}_{14}$ using sound velocity measurements. Phys. Rev. B 96, 134433 (2017).

35. Legrand, W. et al. Room-temperature stabilization of antiferromagnetic skyrmions in synthetic antiferromagnets. Nat. Mater. https://www.nature.com/articles/ s41563-019-0468-3 (2019).

36. Dewhurst, C. D. GRASP user manual. ILL Grenoble, ILL03DE01T (2003).

37. Scagnoli, V. et al. Observation of orbital currents in CuO. Science 332, 696-698 (2011).

\section{ACKNOWLEDGEMENTS}

We thank P.M. Derlet for insightful discussions, K. Rolfs and E. Pomjakushina for their assistance in sample characterization. We acknowledge A. Hadj-Azzem and J. Balay for their help in the preparation of the samples and A.A. Mukhin for fruitful discussions. M.R., N.G., and J.W. acknowledge the financial support of the Swiss National Science Foundation (SNSF) (Sinergia project 'Toroidal moments' No. CRSII2 147606), (project No. 200021_162863) and Sinergia project 'NanoSkyrmionics' (grant CRSII5_171003), respectively. This project has received funding from the European Union Horizon 2020 research and innovation program under grant agreement No 730872 CALIPSOplus. It was also financially supported by Grant No. ANR-13-BS04-0013. E.C. has benefited from a PRESTIGE (Program No. 2014-1-0020) fellowship and CMIRA-Accueil Pro-2015 funding for this research work. Part of this work is based on experiments performed at the Swiss spallation neutron source SINQ, Paul Scherrer Institute, Villigen, Switzerland and at the European neutron source, Institut Laue Langevin, Grenoble, France.

\section{AUTHOR CONTRIBUTIONS}

M.R. and E.C. contributed equally to this work. P. L. synthesized the single-crystals. M. R., N.G., and U.S. performed the RXD experiments with the help of E.S. and E.W; M.R. analyzed the RXD data with the help of U.S. and V.Sc.; E.C. performed the crystal characterization and the pyrocurrent measurements with the help of V.Si., C.C., F.G., and S.d.B.; R.B., V.Si., E.C., and E.R. performed the neutron diffraction measurements; J.
S.W. and M.R. performed the SANS measurements; A.C. and M.M. developed the theoretical aspects; U.S., V.Si., R.B., and A.C. supervised the project. M.R., E.C., U.S., A.C., and V.Si. prepared the figures and wrote the paper with inputs from all authors.

\section{COMPETING INTERESTS}

The authors declare no competing interests.

\section{ADDITIONAL INFORMATION}

Supplementary information is available for this paper at https://doi.org/10.1038/ s41535-019-0199-3.

Correspondence and requests for materials should be addressed to M.R., E.C. or A.C

Reprints and permission information is available at http://www.nature.com/ reprints

Publisher's note Springer Nature remains neutral with regard to jurisdictional claims in published maps and institutional affiliations.

\begin{abstract}
(c) (i)
Open Access This article is licensed under a Creative Commons Attribution 4.0 International License, which permits use, sharing, adaptation, distribution and reproduction in any medium or format, as long as you give appropriate credit to the original author(s) and the source, provide a link to the Creative Commons license, and indicate if changes were made. The images or other third party material in this article are included in the article's Creative Commons license, unless indicated otherwise in a credit line to the material. If material is not included in the article's Creative Commons license and your intended use is not permitted by statutory regulation or exceeds the permitted use, you will need to obtain permission directly from the copyright holder. To view a copy of this license, visit http://creativecommons. org/licenses/by/4.0/.
\end{abstract}

(c) The Author(s) 2019 